Honam Mathematical J. 35 (2013), No. 3, pp. 525-540

http://dx.doi.org/10.5831/HMJ.2013.35.3.525

\title{
INTERVAL-VALUED FUZZY SUBGROUPS AND LEVEL SUBGROUPS
}

\author{
Jeong Gon Lee, Kul Hur and Pyung Ki Lim,**
}

\begin{abstract}
We introduce the concept of level subgroups of an intervalvalued fuzzy subgroup and study some of its properties. These level subgroups in turn play an important role in the characterization of all interval-valued fuzzy subgroup of a prime cyclic group.
\end{abstract}

\section{Introduction}

In 1975, Zadeh[12] suggested the notion of interval-valued fuzzy sets as another generalization of fuzzy sets introduced by himself[11]. After that time, Biswas[1] applied it to group theory, and Gorzalczany[4] suggested a method of inference in approximate reasoning by using intervalvalued fuzzy sets. Moreover Montal and Samanta[10] introduced the concept of topology of interval-valued fuzzy sets and investigate some of its properties and Jun et. al[6] investigated interval-valued fuzzy strong semi-openness and interval-valued fuzzy strong semi-continuity. Recently, Hur et. al[3,9] studied interval-valued smooth topological spaces and interval-valued fuzzy generalized bi-ideals of a semigroup, respectively. Furthermore, Kang and Hur[8] investigated interval-valued fuzzy subgroups and rings, and Kang[7] dealt with problems of the image and preimage of an interval-valued fuzzy set under a homomorphism. In particular, Cheong and Hur[2] studied interval-valued fuzzy ideals and bi-ideals of a semigroup, and Lim et. al[5] investigated interval-valued fuzzy normal subgroups.

In this paper, we obtain a similar characterization of all intervalvalued fuzzy subgroups of finite cyclic groups. For this, we study some

Received July 22, 2013. Accepted July 29, 2013.

2010 Mathematics Subject Classification. 03F55, 20N25.

Key words and phrases. interval-valued fuzzy set, interval-valued fuzzy subgroup, level subgroup.

${ }^{\dagger}$ This paper was supported by Wonkwang University in 2013.

${ }^{*}$ Corresponding author 
properties of level subgroups of an interval-valued fuzzy subgroup in the first part of the paper. These level subgroups in turn play an important role in the above characterization.

\section{Preliminaries}

We will list some concepts and results needed in the later sections. Throughout this paper, we will denote the unit interval $[0,1]$ as $I$.

Let $D(I)$ be the set of all closed subintervals of the unit interval $[0,1]$. The elements of $D(I)$ are generally denoted by capital letters $M, N, \cdots$, and note that $M=\left[M^{L}, M^{U}\right]$, where $M^{L}$ and $M^{U}$ are the lower and the upper end points respectively. Especially, we denote $\mathbf{0}=[0,0], \mathbf{1}$ $=[1,1]$, and $\boldsymbol{a}=[a, a]$ for every $a \in(0,1)$. We also note that

(i) $(\forall M, N \in D(I))\left(M=N \Leftrightarrow M^{L}=N^{L}, M^{U}=N^{U}\right)$,

(ii) $(\forall M, N \in D(I))\left(M=N \leq M^{L} \leq N^{L}, M^{U} \leq N^{U}\right)$.

For every $M \in D(I)$, the complement of $M$, denoted by $M^{C}$, is defined by $M^{C}=1-M=\left[1-M^{U}, 1-M^{L}\right]$ (See [10]).

Definition 2.1. $[4,12]$ A mapping $A: X \rightarrow D(I)$ is called an intervalvalued fuzzy set(in short, IVFS) in $X$, denoted by $A=\left[A^{L}, A^{U}\right]$, if $A^{L}, A^{U} \in I^{X}$ such that $A^{L} \leq A^{U}$, i.e., $A^{L}(x) \leq A^{U}(x)$ for each $x \in X$, where $A^{L}(x)\left[\operatorname{resp} A^{U}(x)\right]$ is called the lower[resp upper] end point of $x$ to $A$. For any $[a, b] \in D(I)$, the interval-valued fuzzy set $A$ in $X$ defined by $A(x)=\left[A^{L}(x), A^{U}(x)\right]=[a, b]$ for each $x \in X$ is denoted by $\left.\widetilde{[a, b}\right]$ and if $a=b$, then the IVFS $\widetilde{[a, b}]$ is denoted by simply $\widetilde{a}$. In particular, $\widetilde{0}$ and $\widetilde{1}$ denote the interval-valued fuzzy empty set and the interval-valued fuzzy whole set in $X$, respectively.

We will denote the set of all IVFSs in $X$ as $D(I)^{X}$. It is clear that set $A=[A, A] \in D(I)^{X}$ for each $A \in I^{X}$.

Definition 2.2.[10] Let $A, B \in D(I)^{X}$ and let $\left\{A_{\alpha}\right\}_{\alpha \in \Gamma} \subset D(I)^{X}$. Then

(i) $A \subset B$ iff $A^{L} \leq B^{L}$ and $A^{U} \leq B^{U}$.

(ii) $A=B$ iff $A \subset B$ and $B \subset A$.

(iii) $A^{C}=\left[1-A^{U}, 1-A^{L}\right]$.

(iv) $A \cup B=\left[A^{L} \vee B^{L}, A^{U} \vee B^{U}\right]$.

(iv) $\bigcup_{\alpha \in \Gamma} A_{\alpha}=\left[\bigvee_{\alpha \in \Gamma} A_{\alpha}^{L}, \bigvee_{\alpha \in \Gamma} A_{\alpha}^{U}\right]$. 
(v) $A \cap B=\left[A^{L} \wedge B^{L}, A^{U} \wedge B^{U}\right]$.

$(\mathrm{v})^{\prime} \bigcap_{\alpha \in \Gamma} A_{\alpha}=\left[\bigwedge_{\alpha \in \Gamma} A_{\alpha}^{L}, \bigwedge_{\alpha \in \Gamma} A_{\alpha}^{U}\right]$

Definition 2.3. [8] Let $A$ be an IVFS in a set $X$ and let $[\lambda, \mu] \in D(I)$. Then the set $A^{[\lambda, \mu]}=\left\{x \in X: A^{L}(x) \geq \lambda\right.$ and $\left.A^{U}(x) \geq \mu\right\}$ is called a $[\lambda, \mu]$-level subset of $A$.

The following is the immediate result of Definition 2.3.

Proposition 2.4. Let $A$ be an IVFS in a set $X$ and let $\left[\lambda_{1}, \mu_{1}\right],\left[\lambda_{2}, \mu_{2}\right] \in$ ImA. If $\lambda_{1} \leq \lambda_{2}$ and $\mu_{1} \leq \mu_{2}$, then $A^{\left[\lambda_{2}, \mu_{2}\right]} \subset A^{\left[\lambda_{1}, \mu_{1}\right]}$.

Definition 2.5. [8] Let $G$ be a group and let $A \in D(I)^{G}$. Then $A$ is called an interval-valued fuzzy subgroup (in short, $I V G$ ) of $G$ if it satisfies the following conditions:

(i) $A^{L}(x y) \geq A^{L}(x) \wedge A^{L}(y)$ and $A^{U}(x y) \geq A^{U}(x) \wedge A^{U}(y)$ for each $x, y \in G$.

(ii) $A^{L}\left(x^{-1}\right) \geq A^{L}(x)$ and $A^{U}\left(x^{-1}\right) \geq A^{U}(x)$ for each $x \in G$.

We will denote the set of all IVGs of $G$ as $\operatorname{IVG}(G)$.

Result 2.A.[1, Proposition 3.1] Let $A$ be an IVG of a group $G$. Then

(a) $A\left(x^{-1}\right)=A(x)$.

(b) $A^{L}(x) \leq A^{L}(e)$ and $A^{U}(x) \leq A^{U}(e)$ for each $x \in G$, where $e$ is the identity element of $G$.

Result 2.B.[8, Propositions 4.16 and 4.17] Let $A$ be an IVFS of a group $G$. Then $A \in \operatorname{IVG}(G)$ if and only if for each $[\lambda, \mu] \in D(I)$ with $\lambda \leq A^{L}(e)$ and $\mu \leq A^{U}(e), A^{[\lambda, \mu]}$ is a subgroup of $G$.

Result 2.C. [8, Proposition 4.12] Let $G_{p}$ be the cyclic group of prime order $p$. Then $A \in \operatorname{IVG}\left(G_{p}\right)$ if and only if $A^{L}(x)=A^{L}(1) \leq A^{L}(0)$ and $A^{U}(x)=A^{U}(1) \leq A^{U}(0)$ for any $0 \neq x \in G_{p}$.

\section{Level subgroups}

From Result 2.B, we define the following concept. 
Definition 3.1. Let $G$ be a group and let $A \in \operatorname{IVG}(G)$ and let $[\lambda, \mu] \in$ $D(I)$ such that $\lambda \leq A^{L}(e)$ and $\mu \leq A^{U}(e)$. Then $A^{[\lambda, \mu]}$ is called a $[\lambda, \mu]$-level subgroup of $A$.

Let $G$ be a finite group. Then the number of subgroups of $G$ is finite. However, the number of level subgroups of an IVG $A$ appears to be infinite. Indeed, since every level subgroup is a subgroup of $G$, not all these level subgroups are distinct.

Example 3.2. Let $G$ be the Klein four-group:

$$
G=\left\{a, b, a^{2}=b^{2}=(a b)^{2}=e\right\} .
$$

Then the elements of $G$ are $e, a, b$ and $a b$. Moreover, it is clear that the number of subgroups of $G$ is finite. We define a mapping $A=: G \rightarrow D(I)$ as follows :

$$
A(e)=\left[\lambda_{0}, \mu_{0}\right], A(a)=\left[\lambda_{1}, \mu_{1}\right], A(b)=\left[\lambda_{2}, \mu_{2}\right] \text { and } A(a b)=\left[\lambda_{3}, \mu_{3}\right],
$$

where $\left[\lambda_{i}, \mu_{i}\right] \in D(I)(i=0,1,2$ and 3$), \lambda_{0} \geq \lambda_{i}, \mu_{0} \geq \mu_{i}(i=1,2,3)$ and $\lambda_{3} \geq \lambda_{1} \wedge \lambda_{2}, \mu_{3} \geq \mu_{1} \wedge \mu_{2}$. Then we can easily see that $A \in \operatorname{IVG}(G)$. Consider the family $\mathcal{P}=\left\{A^{[\lambda, \mu]}:[\lambda, \mu] \in D(I), \lambda \leq A^{L}(e)\right.$ and $\mu \leq$ $\left.A^{U}(e)\right\}$. Then, by Result 2.B, $\mathcal{P}$ is a family of level subgroups of $G$. Furthermore, $\mathcal{P}$ is infinite. But we can see that all members of $\mathcal{P}$ are not distinct.

Proposition 3.3. Let $G$ be a group and let $A \in \operatorname{IVG}(G)$. Two level subgroups $A^{\left[t_{1}, s_{1}\right]}$ and $A^{\left[t_{2}, s_{2}\right]}$ (with $t_{1}<t_{2}$ and $s_{1}<s_{2}$ ) of $A$ are equal if and only if there is no $x \in G$ such that $t_{1}<A^{L}(x)<t_{2}$ and $s_{1}<A^{U}(x)<s_{2}$.

Proof. $(\Rightarrow)$ : Suppose $A^{\left[t_{1}, s_{1}\right]}=A^{\left[t_{2}, s_{2}\right]}$. Assume that there exists an $x \in G$ such that $t_{1}<A^{L}(x)<t_{2}$ and $s_{1}<A^{U}(x)<s_{2}$. Then $x \in$ $A^{\left[t_{1}, s_{1}\right]}$ and $x \notin A^{\left[t_{2}, s_{2}\right]}$. Thus, by Proposition 2.4, $A^{\left[t_{2}, s_{2}\right]} \varsubsetneqq A^{\left[t_{1}, s_{1}\right]}$. This contradicts the hypothesis.

$(\Leftarrow)$ : Suppose the necessary condition holds. Since $t_{1}<t_{2}$ and $s_{1}<s_{2}$, by Proposition 2.4, $A^{\left[t_{2}, s_{2}\right]} \subset A^{\left[t_{1}, s_{1}\right]}$. Let $x \in A^{\left[t_{1}, s_{1}\right]}$. Then $t_{1} \leq A^{L}(x)$ and $s_{1} \leq A^{U}(x)$. By the hypothesis, $t_{2} \leq A^{L}(x)$ and $s_{2} \leq A^{U}(x)$. Thus $x \in A^{\left[t_{2}, s_{2}\right]}$. So $A^{\left[t_{1}, s_{1}\right]} \subset A^{\left[t_{2}, s_{2}\right]}$. Hence $A^{\left[t_{1}, s_{1}\right]}=A^{\left[t_{2}, s_{2}\right]}$. 
Corollary 3.4. Let $G$ be a finite group of order $n$ and let $A \in \operatorname{IVG}(G)$. Let $\operatorname{ImA}=\left\{\left[t_{i}, s_{i}\right]\right): A(x)=\left[t_{i}, s_{i}\right]$ for some $\left.x \in G\right\}$. Then $\left\{A^{\left[t_{i}, s_{i}\right]}\right\}$ is the set of the only level subgroups of $A$.

Proof. By Result 2.B, $A^{\left[t_{i}, s_{i}\right]}$ is a subgroup of $G$. Let $[\lambda, \mu] \in D(I)$ such that $[\lambda, \mu] \notin \operatorname{ImA}$.

Case(i) : Suppose $t_{i}<\lambda<t_{j}$ and $s_{i}<\mu>s_{j}$, where $\left[t_{i}, s_{i}\right],\left[t_{j}, s_{j}\right] \in$ ImA. Then, by Proposition 3.3, $A^{\left[t_{i}, s_{i}\right]}=A^{\left[t_{j}, s_{j}\right]}=A^{[\lambda, \mu]}$.

Case(ii) : Suppose $\lambda<t_{r}$ and $\mu<s_{r}$, where $\left[t_{r}, s_{r}\right]$ is the least element in ImA. Then, by Proposition 3.3, $A^{\left[t_{r}, s_{r}\right]}=G=A^{[\lambda, \mu]}$.

Case(iii) : Suppose $t_{0}<\lambda$ and $s_{0}<\mu$, where $\left[t_{0}, s_{0}\right]$ is the greast element of ImA. Then, by Proposition 3.3, $A^{[\lambda, \mu]}=A^{\left[t_{i}, s_{i}\right]}=\{e\}$.

Hence, in any cases, for each $[\lambda, \mu] \in D(I)$, the $[\lambda, \mu]$-level subgroup is one of $\left\{A^{\left[t_{i}, s_{i}\right]}\right\}$, where $\left[t_{i}, s_{i}\right] \in \operatorname{ImA}$.

Proposition 3.5. Any subgroup $H$ of a group $G$ can be realized as a level subgroup of some IVG of $G$.

Proof. We define a mapping $A: G \rightarrow D(I)$ as follows: For each $x \in G$, $A(x)=[t, s]$ if $x \in H$ and $A(x)=[0,0]$ if $x \notin H$, where $[t, s] \in D(I)$. Let $x, y \in G$.

Case (i) : Suppose $x, y \in H$. Then $x y \in H$. Thus

$$
A^{L}(x y)=A^{L}(x)=A^{L}(y)=t \text { and } A^{U}(x y)=A^{U}(x)=A^{U}(y)=s .
$$

So

$$
A^{L}(x y) \geq A^{L}(x) \wedge A^{L}(y) \text { and } A^{U}(x y) \geq A^{U}(x) \wedge A^{U}(y) .
$$

Since $x \in H, x^{-1} \in H$. Thus $A^{L}\left(x^{-1}\right)=t$ and $A^{U}\left(x^{-1}\right)=s$. Hence $A^{L}\left(x^{-1}\right) \geq A^{L}(x)$ and $A^{U}\left(x^{-1}\right) \leq A^{U}(x)$.

Case (ii) : Suppose $x \in H$ and $y \notin H$. Then $x y \notin H$. Thus

$A^{L}(x)=t, A^{L}(y)=A^{L}(x y)=0$ and $A^{U}(x)=s, A^{U}(y)=A^{U}(x y)=0$.

So

$$
A^{L}(x y) \geq A^{L}(x) \wedge A^{L}(y) \text { and } A^{U}(x y) \geq A^{U}(x) \wedge A^{U}(y) .
$$

Also, we have

$$
A^{L}\left(x^{-1}\right) \geq A^{L}(x) \text { and } A^{U}\left(x^{-1}\right) \geq A^{U}(x) .
$$

Case (iii) : Suppose $x \notin H$ and $y \in H$. Then, we have the same ones as results of case (ii). 
Case (iv) : Suppose $x \notin H$ and $y \notin H$. Then $x y$ may or may not belong to $H$. In any case, we have

$$
A^{L}(x y) \geq A^{L}(x) \wedge A^{L}(y), A^{U}(x y) \geq A^{U}(x) \wedge A^{U}(y)
$$

and

$$
A^{L}\left(x^{-1}\right) \geq A^{L}(x), A^{U}\left(x^{-1}\right) \geq A^{U}(x) .
$$

Hence, in all cases, $A \in \operatorname{IVG}(G)$. In fact, $H=A^{[t, s]}$. This completes the proof.

The following result is the generalization of Proposition 3.5.

Proposition 3.6. Let $G$ be a group and let the following be any chain of subgroups

$$
G_{0} \subset G_{1} \subset \cdots \subset G_{r}=G .
$$

Then there exists an interval-valued fuzzy subgroup of $G$ whose level subgroups are precisely the members of this chain.

Proof. Consider the following set of real numbers:

$$
t_{0}>t_{1}>\cdots>t_{r} \text { and } s_{0}>s_{1}>\cdots>s_{r},
$$

where $\left[t_{i}, s_{i}\right] \in D(I)$ for each $i$. We define a mapping $A: G \rightarrow D(I)$ as follows:

$$
A\left(G_{0}\right)=\left[t_{0}, s_{0}\right] \text { and } A\left(\hat{G}_{i}\right)=\left[t_{i}, s_{i}\right],
$$

where $\hat{G}_{i}=G_{i} \backslash G_{i-1}$ for $i=1,2, \cdots, r$. Let $x, y \in G$.

Case (i) : Suppose $x, y \in \hat{G}_{i}$. Then $A(x)=\left[t_{i}, s_{i}\right]=A(y)$. Since $G_{i}$ is a subgroup, $x y \in G_{i}$. Thus either $x y \in G_{i}$ or $x y \in G_{i-1}$. In any case,

$$
A^{L}(x y) \geq t_{i}=A^{L}(x) \wedge A^{L}(y) \text { and } A^{U}(x y) \geq s_{i}=A^{U}(x) \wedge A^{U}(y) .
$$

On the other hand, $x^{-1} \in G_{i}$. Thus

$$
A^{L}\left(x^{-1}\right) \geq t_{i}=A^{L}(x) \text { and } A^{U}\left(x^{-1}\right) \geq s_{i}=A^{U}(x) .
$$

Case (ii): Suppose $x \in \hat{G}_{i}, y \in \hat{G}_{j}$ and $i>j$. Then $A(x)=\left[t_{i}, s_{i}\right]$ and $A(y)=\left[t_{j}, s_{j}\right]$. Since $G_{j} \subset G_{i}$ and $G_{i}$ is a subgroup, $x y \in G_{i}$. Thus

$$
A^{L}(x y) \geq t_{i}=A^{L}(x) \wedge A^{L}(y) \text { and } A^{U}(x y) \geq s_{i}=A^{U}(x) \wedge A^{U}(y) .
$$

On the other hand, $x^{-1} \in C_{i}$. Thus

$$
A^{L}\left(x^{-1}\right) \geq t_{i}=A^{L}(x) \text { and } A^{U}\left(x^{-1}\right) \geq s_{i}=A^{U}(x) .
$$

So, in either case, we can see that $A \in \operatorname{IVG}(G)$. 
Now, from the definition of $A, \operatorname{ImA}=\left\{\left[t_{0}, s_{0}\right], \cdots,\left[t_{r}, s_{r}\right]\right\}$. Thus the level subgroups of $A$ are given by the chain of subgroups

$$
A^{\left[t_{0}, s_{0}\right]} \subset A^{\left[t_{1}, s_{1}\right]} \subset \cdots \subset A^{\left[t_{r}, s_{r}\right]}=G .
$$

We claim that $A^{\left[t_{i}, s_{i}\right]}=G_{i}, 0<i \leq r$. By the definitions of $A$ and $A^{\left[t_{i}, s_{i}\right]}$, it is clear that $G_{i} \subset A^{\left[t_{i}, s_{i}\right]}$. Let $x \in A^{\left[t_{i}, s_{i}\right]}$. Then

$$
A^{L}(x) \geq t_{i} \text { and } A^{U}(x) \geq s_{i} .
$$

Thus $x \notin G_{j}$ for $j>i$. So $A(x) \in\left\{\left[t_{1}, s_{1}\right], \cdots,\left[t_{i}, s_{i}\right]\right\}$, i.e., $x \in G_{k}$ for some $k \leq i$. Since $G_{k} \subset G_{i}, x \in G_{i}$. Thus $A^{\left[t_{i}, s_{i}\right]} \subset G_{i}$. Hence $A^{\left[t_{i}, s_{i}\right]}=G_{i}, 0 \leq i \leq r$. This completes the proof.

As a consequence of Proposition 3.6, the level subgroups of an IVG $A$ form a chain. Since

$$
A^{L}(x) \leq A^{L}(e) \text { and } A^{U}(x) \leq A^{U}(e)
$$

for each $x \in G, A^{\left[t_{0}, s_{0}\right]}$ is the smallest level subgroup of $A$, where $A(e)=\left[t_{0}, s_{0}\right]$. Thus we have the chain

$$
(e)=A^{\left[t_{0}, s_{0}\right]} \subset A^{\left[t_{1}, s_{1}\right]} \subset \cdots \subset A^{\left[t_{r}, s_{r}\right]}=G,
$$

where $t_{0}>t_{1}>\cdots>t_{r}$ and $s_{0}>s_{1}>\cdots>s_{r}$. We denote this chain (3.1) of level subgroups by $C(A)$. In general, as all the subgroups of $G$ do not form a chain, it follows that not all subgroups of $G$ are level subgroups of a given interval-valued fuzzy subgroup. So it is an interesting problem to find an IVG $A$ of $G$ which accommodates as many subgroups of $G$ as possible in $C(A)$.

Proposition 3.7. Let $G$ be a finite group such that $G=G_{p_{1}} \times G_{p_{2}} \times$ $\cdots \times G_{p_{r}}$, where the $G_{p_{i}}$ are prime cyclic groups of orders $p_{i}$. Then there exists an $A \in \operatorname{IVG}(G)$ such that $C(A)$ is a maximal chain of length $r+1$.

Proof. We prove by induction on $r$. Suppose $r=1$. Then $G=C_{p_{1}}$. Then, by Result 2.C, there exists an $A \in \operatorname{IVG}(G)$ such that $A(e)=$ $\left[t_{0}, s_{0}\right], A(x)=\left[t_{1}, s_{1}\right]$ for each $e \neq x \in G$ and $t_{1}<t_{0}$ and $s_{1}<s_{0}$. Thus $A^{\left[t_{0}, s_{0}\right]}=(e)$ and $A^{\left[t_{1}, s_{1}\right]}=G$. So $A^{\left[t_{0}, s_{0}\right]} \subset A^{\left[t_{1}, s_{1}\right]}$ is the maximal chain and of length 2. Hence the theorem is true for $r=1$.

Now let $r>1$ and suppose the theorem is true for the integers $\leq r-1$. Let $H=G_{p_{1}} \times G_{p_{2}} \times \cdots \times G_{p_{r-1}}$. Then $G=H \times G_{p_{r}}$. Define the mapping $A: G \longrightarrow D(I)$ as follows :

$$
A(e)=\left[t_{0}, s_{0}\right],
$$




$$
\begin{gathered}
\widehat{A\left(G_{p_{1}}\right)}=\left[t_{1}, s_{1}\right], \\
A\left(G_{p_{1} \times G_{p_{2}}}\right)=\left[t_{2}, s_{2}\right], \\
\cdots \cdots \cdots \cdots \cdots \cdots \cdots, \\
A\left(\widehat{H \times G_{p_{r}}}\right)=\left[t_{r}, s_{r}\right],
\end{gathered}
$$

where $t_{0}>t_{1}>t_{2}>\cdots>t_{r}, s_{0}>s_{1}>s_{2}<\cdots>s_{r}, t_{i} \leq s_{i}$ and $\widehat{G_{p_{1}}}=G_{p_{1}} \backslash(e), \widehat{G_{p_{1}} \times G_{p_{2}}}=G_{p_{1}} \times G_{p_{2}} \backslash G_{p_{1}}$, and so on. We will show that $A \in \operatorname{IVG}(G)$. Let $x, y \in G$.

Case (i): Suppose $x, y \in H$. Then $x y \in H$. By the induction,

$$
A^{L}(x y) \geq A^{L}(x) \wedge A^{L}(y), A^{U}(x y) \geq A^{U}(x) \wedge A^{U}(y)
$$

and

$$
A^{L}\left(x^{-1}\right) \geq A^{L}(x), A^{U}\left(x^{-1}\right) \geq A^{U}(x) .
$$

Case (ii): Suppose $x \in H$ and $y \in G \backslash H$. Then $x y \notin H$. Thus $A(x y)=\left[t_{r}, s_{r}\right], A^{L}(x) \geq t_{r-1}, A^{U}(x) \geq s_{r-1}$ and $A(y)=\left[t_{r}, s_{r}\right]$. So

$$
A^{L}(x y) \geq A^{L}(x) \wedge A^{L}(y), A^{U}(x y) \geq A^{U}(x) \wedge A^{U}(y)
$$

and

$$
A^{L}\left(x^{-1}\right) \geq A^{L}(x), A^{U}\left(x^{-1}\right) \geq A^{U}(x) .
$$

Case (iii): Suppose $x \in G \backslash H$ and $y \in H$. Then, we have the same ones as the results of case(ii).

Case (iv): Suppose $x \notin H$ and $y \notin H$. Then also we can easily see that

$$
A^{L}(x y) \geq A^{L}(x) \wedge A^{L}(y), A^{U}(x y) \geq A^{U}(x) \wedge A^{U}(y)
$$

and

$$
A^{L}\left(x^{-1}\right) \geq A^{L}(x), A^{U}\left(x^{-1}\right) \geq A^{U}(x) .
$$

So, in either cases, $A \in \operatorname{IVG}(G)$. Moreover,

$$
\begin{gathered}
A^{\left[t_{0}, s_{0}\right]}=(e), \\
A^{\left[t_{1}, s_{1}\right]}=G_{r_{1}}, \\
A^{\left[t_{2}, s_{2}\right]}=G_{r_{1}} \times G_{r_{2}}, \\
\cdots \ldots \ldots \cdots \cdots \cdots, \\
A^{\left[t_{r}, s_{r}\right]}=H \times G_{r_{r}} .
\end{gathered}
$$

Hence $A^{\left[t_{0}, s_{0}\right]} \subset A^{\left[t_{1}, s_{1}\right]} \subset \cdots \subset A^{\left[t_{r}, s_{r}\right]}$ is $C(A)$ which is maximal and of length $r+1$. This completes the proof.

Remark 3.8. In the same way, we can find an IVG $A$ with the maximal $C(A)$ in the following cases :

(i) $G$ is a cyclic $p$-group. 
(ii) $G$ is the direct product of cyclic $p$-group.

(iii) $G$ is a finite abelian group.

We can easily check these cases by adopting the same technique as proof in Proposition 3.7.

In the following example, we show that two interval-valued fuzzy subgroups of a group may have an identical family of level subgroups but the interval-valued fuzzy subgroups may not be equal.

Example 3.9. Consider the Klein four-group $G$ given in Example 3.2. Let $\left[t_{i}, s_{i}\right] \in D(I)$ such that $t_{0}>t_{1}>t_{2}, s_{0}>s_{1}>s_{2}$, where $i=0,1,2$. We define a mapping $A: G \rightarrow D(I)$ as follows :

$$
A(e)=\left[t_{0}, s_{0}\right], A(a)=\left[t_{1}, s_{1}\right], A(b)=\left[t_{2}, s_{2}\right], A(a b)=\left[t_{2}, s_{2}\right] .
$$

Then clearly $A \in \operatorname{IVG}(G)$ and $\operatorname{ImA}=\left\{\left[t_{0}, s_{0}\right],\left[t_{1}, s_{1}\right],\left[t_{2}, s_{2}\right]\right\}$. Moreover, the level subgroups of $A$ are

$$
A^{\left[t_{0}, s_{0}\right]}=\{e\}, A^{\left[t_{1}, s_{1}\right]}=\{e, a\}, A^{\left[t_{2}, s_{2}\right]}=G .
$$

Now let $\left[\lambda_{i}, \mu_{i}\right] \in D(I)$ such that $\lambda_{0}>\lambda_{1}>\lambda_{2}, \mu_{0}>\mu_{1}>\mu_{2}$, for $i=0,1,2$ and $\left\{\left[t_{0}, s_{0}\right],\left[t_{1}, s_{1}\right],\left[t_{2}, s_{2}\right]\right\} \cap\left\{\left[\lambda_{0}, \mu_{0}\right],\left[\lambda_{1}, \mu_{1}\right],\left[\lambda_{2}, \mu_{2}\right]\right\}=\phi$. We define a mapping $B: G \rightarrow D(I)$ as follows :

$$
B(e)=\left[\lambda_{0}, \mu_{0}\right], B(a)=\left[\lambda_{1}, \mu_{1}\right], B(b)=\left[\lambda_{2}, \mu_{2}\right], B(a b)=\left[\lambda_{2}, \mu_{2}\right] .
$$

Then clearly $B \in \operatorname{IVG}(G)$. Moreover, the level subgroups of $B$ are

$$
B^{\left[\lambda_{0}, \mu_{0}\right]}=\{e\}, B^{\left[\lambda_{1}, \mu_{1}\right]}=\{e, a\}, B^{\left[\lambda_{2}, \mu_{2}\right]}=G .
$$

Hence $A$ and $B$ have the same family of level subgroups but $A \neq B$.

The following is the immediate result of Definition 2.3.

Lemma 3.10. Let $G$ be a finite group and let $A \in \operatorname{IVG}(G)$. If $\left[t_{i}, s_{i}\right],\left[t_{j}, s_{j}\right] \in \operatorname{ImA}$ such that $A^{\left[t_{i}, s_{i}\right]}=A^{\left[t_{j}, s_{j}\right]}$, then $\left[t_{i}, s_{i}\right]=\left[t_{j}, s_{j}\right]$.

Proposition 3.11 Let $G$ be a finite group and let $A, B \in \operatorname{IVG}(G)$ with identical family of level subgroups. If $\operatorname{ImA}=\left\{\left[t_{0}, s_{0}\right], \cdots,\left[t_{r}, s_{r}\right]\right\}$ and $\operatorname{ImB}=\left\{\left[\lambda_{0}, \mu_{0}\right], \cdots,\left[\lambda_{k}, \mu_{k}\right]\right\}$, where $t_{0}>\cdots>t_{r}, s_{0}>\cdots>s_{r}$ and $\lambda_{0}>\cdots>\lambda_{k}, \mu_{0}>\cdots>\mu_{k}$, then we have

(a) $r=k$.

(b) $A^{\left[t_{i}, s_{i}\right]}=B^{\left[\lambda_{i}, \mu_{i}\right]}, 0 \leq i \leq r$. 
(c) if $x \in G$ such that $A(x)=\left[t_{i}, s_{i}\right]$, then $B(x)=\left[\lambda_{i}, \mu_{i}\right], 0 \leq i \leq r$.

Proof. (a) By Corollary 3.4, the only level subgroups of $A$ and $B$ are the two families $\left\{A^{\left[t_{i}, s_{i}\right]}\right\}$ and $\left\{B^{\left[\lambda_{i}, \mu_{i}\right]}\right\}$. Hence, by hypothesis, $r=k$.

(b) By $(a)$ and Corollary 3.3, there exist two chains of level subgroups

$$
A^{\left[t_{0}, s_{0}\right]} \subset A^{\left[t_{1}, s_{1}\right]} \subset \cdots \subset A^{\left[t_{r}, s_{r}\right]}=G
$$

and

$$
B^{\left[\lambda_{0}, \mu_{0}\right]} \subset B^{\left[\lambda_{1}, \mu_{1}\right]} \subset \cdots \subset B^{\left[\lambda_{k}, \mu_{k}\right]}=G .
$$

From Proposition 2.4, it follows clearly that

Suppose $\left[t_{i}, s_{i}\right],\left[t_{j}, s_{j}\right] \in \operatorname{ImA}$ such that $t_{i}>t_{j}$ and $s_{i}>s_{j}$. Then

$$
A^{\left[t_{i}, s_{i}\right]} \subset A^{\left[t_{j}, s_{j}\right]} \text {. (3.2) }
$$

Suppose $\left[\lambda_{i}, \mu_{i}\right],\left[\lambda_{j}, \mu_{j}\right] \in \operatorname{ImB}$ such that $\lambda_{i}>\lambda_{j}$ and $\mu_{i}>\mu_{j}$. Then

$$
B^{\left[\lambda_{i}, \mu_{i}\right]} \subset B^{\left[\lambda_{j}, \mu_{j}\right]} .
$$

Since $\left\{A^{\left[t_{i}, s_{i}\right]}\right\}=\left\{B^{\left[\lambda_{i}, \mu_{i}\right]}\right\}$, it is clear that $A^{\left[t_{0}, s_{0}\right]}=B^{\left[\lambda_{0}, \mu_{0}\right]}$. Now by hypothesis, $A^{\left[t_{1}, s_{1}\right]}=B^{\left[\lambda_{j}, \mu_{j}\right]}$ for some $j>0$. Assume that $A^{\left[t_{1}, s_{1}\right]}=$ $B^{\left[\lambda_{j}, \mu_{j}\right]}$ for some $j>1$. Again, we have that $B^{\left[\lambda_{1}, \mu_{1}\right]}=A^{\left[t_{i}, s_{i}\right]}$ for some $t_{i}>t_{1}$ and $s_{i}>s_{1}$. It is clear that $\left[t_{i}, s_{i}\right]=\left[t_{1}, s_{1}\right]$. Thus, by (3.2),

$$
A^{\left[t_{i}, s_{i}\right]}=B^{\left[\lambda_{1}, \mu_{1}\right]} \subset B^{\left[\lambda_{j}, \mu_{j}\right]} .
$$

Also, by (3.3),

$$
B^{\left[\lambda_{j}, \mu_{j}\right]}=A^{\left[t_{1}, s_{1}\right]} \subset A^{\left[t_{i}, s_{i}\right]} .
$$

However, (3.4) and (3.5) contradict one another as inclusions are both proper inclusions. So, we must have that

$$
A^{\left[t_{1}, s_{1}\right]}=B^{\left[\lambda_{1}, \mu_{1}\right]} \text {. }
$$

The rest of the proof follows by induction on $i$ by using arguments exactly on the same lines as above. Hence $A^{\left[t_{i}, s_{i}\right]}=B^{\left[\lambda_{j}, \mu_{j}\right]}, 0 \leq i \leq r$.

(c) Let $x \in G$ such that $A(x)=\left[t_{i}, s_{i}\right]$ and $B(x)=\left[\lambda_{j}, \mu_{j}\right]$. Then, by $(b), A^{\left[t_{i}, s_{i}\right]}=B^{\left[\lambda_{i}, \mu_{i}\right]}$. Since $x \in A^{\left[t_{i}, s_{i}\right]}, x \in B^{\left[\lambda_{i}, \mu_{i}\right]}$. Thus $B(x)=$ $\left[\lambda_{j}, \mu_{j}\right]$, where $\lambda_{j} \geq \lambda_{i}$ and $\mu_{j} \geq \mu_{i}$. By (3.3), $B^{\left[\lambda_{j}, \mu_{j}\right]} \subset B^{\left[\lambda_{i}, \mu_{i}\right]}$. $\operatorname{By}(b), B^{\left[\lambda_{j}, \mu_{j}\right]}=A^{\left[t_{j}, s_{j}\right]}$. Since $x \in B^{\left[\lambda_{j}, \mu_{j}\right]}, x \in A^{\left[t_{j}, s_{j}\right]}$. Thus $A(x)=\left[t_{i}, s_{i}\right]$, where $t_{i} \geq t_{j}$ and $s_{i} \geq s_{j}$. By (3.2), $A^{\left[t_{i}, s_{i}\right]} \subset A^{\left[t_{j}, s_{j}\right]}$. On the other hood, by $(b), A^{\left[t_{i}, s_{i}\right]}=B^{\left[\lambda_{i}, \mu_{i}\right]}$ and $A^{\left[t_{j}, s_{j}\right]}=B^{\left[\lambda_{j}, \mu_{j}\right]}$. Consequently, we have that $B^{\left[\lambda_{i}, \mu_{i}\right]} \subset B^{\left[\lambda_{j}, \mu_{j}\right]}$. So $B^{\left[\lambda_{i}, \mu_{i}\right]}=B^{\left[\lambda_{j}, \mu_{j}\right]}$. Hence, by Lemma 3.10, $\left[\lambda_{i}, \mu_{i}\right]=\left[\lambda_{j}, \mu_{j}\right]$. This completes the proof. 
Theorem 3.12. Let $A, B$ be two IVGs of a finite group $G$ such that the families of level subgroups of $A$ and $B$ are identical. Then $A=B$ if and only if $\operatorname{Im} A=\operatorname{Im} B$.

Proof. $(\Rightarrow)$ : It is obvious.

$(\Leftarrow)$ : Suppose $\operatorname{Im} A=\operatorname{Im} B$. Let $\operatorname{Im} A=\left\{\left[t_{0}, s_{0}\right], \cdots,\left[t_{r}, s_{r}\right]\right\}$ and let $\operatorname{Im} B=\left\{\left[\lambda_{0}, \mu_{0}\right], \cdots,\left[\lambda_{r}, \mu_{r}\right]\right\}$ such that $t_{0}>\cdots>t_{r}, s_{0}>\cdots>s_{r}$ and $\lambda_{0}>\cdots>\lambda_{r}, \mu_{0}>\cdots>\mu_{r}$. Since $\left[\lambda_{0}, \mu_{0}\right] \in \operatorname{Im} B$ and $\operatorname{Im} A=\operatorname{Im} B$, $\left[\lambda_{0}, \mu_{0}\right]=\left[t_{k_{0}}, s_{k_{0}}\right]$ for some $k_{0}$. Suppose $\left[t_{k_{0}}, s_{k_{0}}\right] \neq\left[t_{0}, s_{0}\right]$. Then $t_{k_{0}}<$ $t_{0}$ and $s_{k_{0}}<s_{0}$. Since $\left[\lambda_{1}, \mu_{1}\right] \in \operatorname{ImA},\left[\lambda_{1}, \mu_{1}\right]=\left[t_{k_{1}}, s_{k_{1}}\right]$ for some $k_{1}$. Thus we have

$$
\lambda_{0}>\lambda_{1} \text { and } \mu_{0}>\mu_{1} \text { implies that } t_{k_{0}}>t_{k_{1}} \text { and } s_{k_{0}}>s_{k_{1}} .
$$

By proceeding in this way, we have

$$
t_{k_{0}}>t_{k_{1}}>\cdots>t_{k_{r}} \text { and } s_{k_{0}}>s_{k_{1}}>\cdots>s_{k_{r}} \text {, }
$$

where $\left[\lambda_{0}, \mu_{0}\right]=\left[t_{k_{0}}, s_{k_{0}}\right], t_{k_{0}}>t_{0}$ and $s_{k_{0}}>s_{0}$. They contradict the fact that $\operatorname{ImA}=\operatorname{ImB}$. So we must have that $\left[\lambda_{0}, \mu_{0}\right]=\left[t_{0}, s_{0}\right]$. Arguing in this manner, we obtain that

$$
\left[\lambda_{i}, \mu_{i}\right]=\left[t_{i}, s_{i}\right], 0 \leq i \leq r .
$$

Now let $g_{0}, \cdots, g_{r}$ be distinct elements of $G$ such that $A\left(g_{i}\right)=\left[t_{i}, s_{i}\right], 0 \leq$ $i \leq r$. Then, by Proposition 3.11, $B\left(g_{i}\right)=\left[\lambda_{i}, \mu_{i}\right], 0 \leq i \leq r$. Since $\left[\lambda_{i}, \mu_{i}\right]=\left[t_{i}, s_{i}\right], A(x)=B(x)$ for each $x \in G$. Hence $A=B$. This completes the proof.

The following result is easy to prove.

Lemma 3.13. Let $G$ be a finite group. We define a relation $\sim$ on $\operatorname{IVG}(\mathrm{G})$ as follows : For any $A, B \in \operatorname{IVG}(\mathrm{G}), A \sim B$ if and only of they have an identical family of level subgroups. Then $\sim$ is an equivalence relation on $\operatorname{IVG}(\mathrm{G})$.

We note that by Example 3.9, two elements $A$ and $B$ of IVG(G) may be such that $A \sim B$ but $A$ and $B$ need not be equal.

For each $A \in \operatorname{IVG}(\mathrm{G})$, let $[A]$ denote the equivalence class of $A$. If $G$ is finite, then the number of possible distinct level subgroups of $G$ is finite since each level subgroup is a subgroup of $G$ in the usual sense. By Proposition 3.4, since any subgroup of a group $G$ can be realized as the level subgroup of some interval-valued fuzzy subgroup of $G$, it follows that the number of possible chains of level subgroups is also finite. As 
each equivalence class is characterized completely by its chain of level subgroups, we have the following result.

Corollary 3.14. Let $G$ be a finite group and let $\sim$ be the equivalence relation on $\operatorname{IVG}(\mathrm{G})$ defined by Lemma 3.13. Then $\operatorname{IVG}(G) / \sim$ is finite.

Theorem 3.15. Let $G$ be a finite group and let $\operatorname{LG}(G)=\left\{A^{[\lambda, \mu]}\right.$ : $A^{[\lambda, \mu]}$ is a level subgroup of $G$ and $\left.A \in \operatorname{IVG}(\mathrm{G})\right\}$. Let $\operatorname{SG}(G)$ denote the set of all subgroups of $G$. Then there is a one-to-one correspondence between $\operatorname{SG}(G)$ and $\operatorname{LG}(G) / \sim$, where $\sim$ denote a suitable equivalence relation on $\operatorname{LG}(G)$.

Proof. Let $\sim$ be the equivalence relation on $\operatorname{IVG}(\mathrm{G})$ defined by Lemma 3.13. Then $\operatorname{IVG}(G) / \sim$ is an partition of $G$. Thus

$$
\operatorname{IVG}(\mathrm{G})=\left[S_{1}\right] \cup\left[S_{2}\right] \cup \cdots\left[S_{k}\right],
$$

where $\left[S_{i}\right], 1 \leq i \leq k$, are all distinct equivalence classes. Let us denote

$$
\left\{A_{i}^{\left[t_{j}, s_{j}\right]}: 0 \leq j \leq \lambda_{i} \text { and }\left[t_{j}, s_{j}\right]\right\}
$$

to be the chain of level subgroups associated with the equivalence class $\left[S_{i}\right]$. Then $\operatorname{LG}(G) / \sim$ is a finite set give by

$$
\mathrm{LG}(G) / \sim=\left\{\left[A_{i}^{\left(t_{j}, s_{j}\right)}\right]: 0 \leq j \leq \lambda_{i} \text { and } 1 \leq i \leq k\right\},
$$

where $\left[A_{i}^{\left[t_{j}, s_{j}\right]}\right]$ denotes the equivalence class containing $A_{i}^{\left[\left(t_{j}, s_{j}\right]\right.}$.

From Proposition 3.5, it follows that each subgroup of $G$ is of the form $A_{i}^{\left[t_{j}, s_{j}\right]}$. We define a mapping $f: \operatorname{LG}(G) / \sim \rightarrow \mathrm{SG}(G)$ as follows :

$$
f\left(\left[A_{i}^{\left[t_{j}, s_{j}\right]}\right]\right)=A_{i}^{\left[t_{j}, s_{j}\right]} .
$$

Then we can easily show that $f$ is bijective. This completes the proof.

\section{Characterization of interval-valued fuzzy subgroups of fi- nite cyclic groups}

Proposition 4.1. Let $G$ be a cyclic $p$-group of order $p^{n}$, where $p$ is a prime. Let $A \in \operatorname{IVG}(G)$, let $x, y \in G$ and let $O(x)$ denote the order of $x$.

(a) If $O(x)>O(y)$, then $A^{L}(y) \geq A^{L}(x)$ and $A^{U}(y) \geq A^{U}(x)$.

(b) If $O(x)=O(y)$, then $A(x)=A(y)$. 
Proof. We prove by induction on $n$. Suppose $n=1$. Then $O(G)=p$. Thus the theorem is true by Result 2.A. Let $n>1$ and suppose the theorem is true for all integers $\leq n-1$. Let $H$ be a subgroup of order $p^{n-1}$ and let $x, y \in G$.

Case (i): Suppose $x, y \in H$. Then, by the induction, the results follow.

Case (ii): Suppose $x \notin H$ and $y \in H$. Then $O(x)=p^{n}$ and $O(y)=p^{r}$, where $r \leq n-1$. Thus $x$ is a generator of $G$. So there exists an integer $l$ such that $y=x^{l}$. Hence

$$
A^{L}(y)=A^{L}(x) \wedge \cdots \wedge A^{L}(x)(l \text { times }) \geq A^{L}(x)
$$

and

$$
A^{U}(y)=A^{U}(x) \wedge \cdots \wedge A^{U}(x)(l \text { times }) \geq A^{U}(x)
$$

Case (iii) : Suppose $x \in H$ and $y \notin H$. Then, we have the same ones as the result of case(ii).

Case (iv) : Suppose $x \notin H$ and $y \notin H$. Then $O(x)=O(y)=p^{n}$. Thus $x$ and $y$ are generators of $G$. So there exist integers $l$ and $m$ such that $y=x^{l}$ and $x=y^{m}$. Hence

$$
\begin{aligned}
& A^{L}(x) \geq A^{L}(y) \wedge \cdots \wedge A^{L}(y)(m \text { times }) \geq A^{L}(y), \\
& A^{U}(x) \geq A^{U}(y) \wedge \cdots \wedge A^{U}(y)(m \text { times }) \geq A^{U}(y),
\end{aligned}
$$

and

$$
\begin{aligned}
& A^{L}(y)=A^{L}(x) \wedge \cdots \wedge A^{L}(x)(l \text { times }) \geq A^{L}(x), \\
& A^{U}(y)=A^{U}(x) \wedge \cdots \wedge A^{U}(x)(l \text { times }) \geq A^{U}(x), .
\end{aligned}
$$

Therefore $A(x)=A(y)$. This completes the proof.

Proposition 4.1 is not true in general as shown in the following examples.

Example 4.2. Consider the Kleins 4 - group :

$$
G=\left\{a, b: a^{2}=b^{2}=(a b)^{2}=e\right\} .
$$

We define a mapping $A=\left(\mu_{A}, \nu_{A}\right): G \rightarrow D(I)$ as follows:

$$
A(e)=\left[t_{0}, s_{0}\right], A(a)=\left[t_{1}, s_{1}\right], A(b)=\left[t_{2}, s_{2}\right]=A(a b),
$$

where $\left[t_{0}, s_{0}\right]>\left[t_{1}, s_{1}\right]>\left[t_{2}, s_{2}\right.$ and $\left[t_{i}, s_{i}\right] \in D(I)$ for $i=0,1,2$.

Then clearly $A \in \operatorname{IVG}(G)$. But, even though $O(a)=O(b), A(a) \neq A(b)$. 
For a cyclic group it can be seen that $O(a)=O(b)$ implies $A(a)=$ $A(b)$. But $O(a) \neq O(b)$ may also imply $A(a)=A(b)$.

Example 4.3. Let $G=(a)$ be a cyclic group of order 6 . We define a mapping $A: G \rightarrow D(I)$ as follows:

$A(e)=\left[t_{0}, s_{0}\right], A(a)=A\left(a^{3}\right)=A\left(a^{5}\right)=\left[t_{1}, s_{1}\right], A\left(a^{2}\right)=A\left(a^{4}\right)=\left[t_{2}, s_{2}\right]$, where $\left[t_{0}, s_{0}\right]>\left[t_{1}, s_{1}\right]>\left[t_{2}, s_{2}\right]$ and $\left[t_{i}, s_{i}\right] \in D(I)$ for $i=0,1,2$. Then clearly $A \in I V G(G)$ and $O\left(a^{3}\right) \neq O(a)$. But $A(a)=A\left(a^{3}\right)$.

Now we give the characterization of all IVGs of a finite cyclic group in the following. In fact, the following result is the spacial case of Proposition 3.6.

Theorem 4.4. Let $G$ be a finite cyclic group and let $A \in D(I)^{G}$. Then $A$ is an IVG of $G$ if and only if there exists a maximal chain of subgroups $(e)=G_{0} \subset G_{1} \subset \cdots \subset G_{r}=G$ such that for any $\left[t_{0}, s_{0}\right],\left[t_{1}, s_{1}\right], \cdots,\left[t_{r}, s_{r}\right] \in \operatorname{Im} A$ with $t_{0}>t_{1}>\cdots>t_{r}$ and $s_{0}>s_{1}>$ $\cdots>s_{r}, A(e)=\left[t_{0}, s_{0}\right], A\left(\hat{G}_{1}\right)=\left[t_{1}, s_{1}\right], \cdots, A\left(\hat{G}_{r}\right)=\left[t_{r}, s_{r}\right]$, where $\hat{G}_{i}=G_{i} \backslash G_{i-1}$ for $i=1,2, \cdots, r$.

Proof. $\quad(\Leftarrow)$ : Suppose the necessary condition holds. We define a mapping $A: G \rightarrow D(I)$ by $A(e)=\left[t_{0}, s_{0}\right], A\left(\hat{G}_{1}\right)=\left[t_{1}, s_{1}\right], \cdots, A\left(\hat{G}_{r}\right)=$ $\left[t_{r}, s_{r}\right]$. Let $x, y \in G$.

Case (i) : Suppose $x, y \in G_{i}$ but not in $G_{i-1}$. Then $A(x)=A(y)=$ $\left[t_{i}, s_{i}\right]$ and $x y \in G_{i}$ or $G_{i-1}$. Thus

$$
A^{L}(x y) \geq t_{i}=A^{L}(x) \wedge A^{L}(y)
$$

and

$$
A^{U}(x y) \geq s_{i}=A^{U}(x) \wedge A^{L}(y) .
$$

Moreover, $A^{L}\left(x^{-1}\right) \geq t_{i}=A^{U}(x)$ and $A^{U}\left(x^{-1}\right) \geq s_{i}=A^{U}(x)$.

Case (ii) : Suppose $x \in G_{i}$ but not in $G_{i-1}$ and $y \in G_{j}$ but not $G_{j-1}$, where $i>j$. Then $A(x)=\left[t_{i}, s_{i}\right]$ and $A(y)=\left[t_{j}, s_{j}\right]$. Thus

$$
A^{L}(x y) \geq t_{i}=A^{L}(x) \wedge A^{L}(y)
$$

and

$$
A^{U}(x y) \geq s_{i}=A^{U}(x) \wedge A^{U}(y) .
$$

Also $A^{L}\left(x^{-1}\right) \geq t_{i}=A^{L}(x)$ and $A^{U}\left(x^{-1}\right) \geq s_{i}=A^{U}(x)$. Hence, in all, $A \in \operatorname{IVG}(G)$. 
$(\Rightarrow)$ : Suppose $A \in \operatorname{IVG}(G)$. Then, by Corollary $3.4, A^{\left[t_{0}, s_{0}\right]}, \cdots$, $A^{\left[t_{r}, s_{r}\right]}$ are the only level subgroups of $A$, where $\left\{\left[t_{0}, s_{0}\right],\left[t_{1}, s_{1}\right], \cdots\right.$ $\left.\cdot,\left[t_{r}, s_{r}\right]\right\}=\operatorname{Im} A, t_{0}>t_{1}>\cdots>t_{r}$ and $s_{0}>s_{1}>\cdots>s_{r}$. Furthermore, the level subgroups form a chain $C(A): A^{\left[t_{0}, s_{0}\right]} \subset A^{\left[t_{1}, s_{1}\right]} \subset$ $\cdots \subset A^{\left[t_{r}, s_{r}\right]}$. Thus clearly, $A^{\left[t_{0}, s_{0}\right]}=(e)$ and $A^{\left(t_{r}, s_{r}\right)}=G$.

Suppose $C(A)$ is maximal and let $G_{i}=A^{\left[t_{i}, s_{i}\right]}$. Then the necessary condition holds. Assume that $C(A)$ is not maximal. Then we redefine $C(A)$ by introducing subgroups of $G$. Let us call this chain as

$$
G_{0} \subset G_{1} \subset \cdots \subset G_{s}
$$

where $G_{0}=A^{\left[t_{0}, s_{0}\right]}=(e)$ and $G_{s}=A^{\left[t_{r}, s_{r}\right]}=G$. Then for each $G_{i}$ between $A^{\left[t_{0}, s_{0}\right]}\left(=G_{0}\right)$ and $A^{\left[t_{1}, s_{1}\right]}\left(=G_{j}\right.$ for some $\left.j\right), A\left(\hat{G}_{i}\right)=$ $\left[t_{1}, s_{1}\right]$. Similarly, for each $G_{k}$ between $A^{\left[t_{i}, s_{i}\right]}$ and $A^{\left[t_{i+1}, s_{i+1}\right]}, A\left(\hat{G}_{k}\right)=$ $\left[t_{i+1}, s_{i+1}\right]$ and $A\left(\hat{G}_{s}\right)=\left[t_{r}, s_{r}\right]$. Thus

$$
\begin{gathered}
A\left(\hat{G}_{0}\right)=\left[t_{0}, s_{0}\right], \\
A\left(\hat{G}_{1}\right)=\cdots=A\left(\hat{G}_{j}\right)=\left[t_{1}, s_{1}\right], \\
A\left(\hat{G}_{j+1}\right)=\cdots=A\left(\hat{G}_{k}\right)=\left[t_{r-1}, s_{r-1}\right], \\
\cdots \cdots \cdot \\
A\left(\hat{G}_{s}\right)=\left[t_{r}, s_{r}\right],
\end{gathered}
$$

where $\hat{G}_{1}=G_{1}-G_{0}, \hat{G}_{2}=G_{2}-G_{1}, \cdots, \hat{G}_{s}=G_{s}-G_{s-1}, t_{0}>t_{1}>$ $\cdots>t_{r}$ and $s_{0}>s_{1}>\cdots<s_{r}$. This completes the proof.

The following is the immediate result of Theorem 4.4.

Corollary 4.5. Let $G$ be a cyclic $p$-group of order $p^{r}$ and let $A \in D(I)^{G}$. Then $A \in \operatorname{IVG}(G)$ if and only if for each $x \in G$ with $O(x)=p^{i}, A(x)=$ $\left[t_{i}, s_{i}\right]$, where $i=0,1, \cdots, r, t_{0}>t_{1}>\cdots>t_{r}$ and $s_{0}>s_{1}>\cdots>s_{r}$.

Remark 4.6. We can also prove this Corollary by using Proposition 4.1 .

\section{References}

[1] R. Biswas, Rosenfeld's fuzzy subgroups with interval-valued membership functions, Fuzzy set and systems 63 (1995), 87-90.

[2] M. Cheong and K. Hur, Interval-valued fuzzy ideals and bi-ideals of a semi-group, IJFIS 11 (2011), 259-266.

[3] J. Y. Choi, S. R. Kim and K. Hur, Interval-valued smooth topological spaces, Honam Math. J. 32(4) (2010), 711-738. 
[4] M. B. Gorzalczany, A method of inference in approximate reasoning based on interval-valued fuzzy sets, Fuzzy sets and Systems 21 (1987), 1-17.

[5] S. Y. Jang, K. Hur and P. K. Lim, Interval-valued fuzzy normal subgroups, IJFIS 12(3) (2012), 205-214.

[6] Y. B. Jun, J. J. Bae, S. H. Cho and C. S. Kim, Interval-valued fuzzy strong semi-openness and interval-valued fuzzy strong semi-continuity, Honam Math. J. 28(3) (2006), 417-431.

[7] H. Kang, Interval-valued fuzzy subgroups and homomorphisms, Honam Math. J. 33(4) (2011), 499-518.

[8] H. Kang and K.Hur, Interval-valued fuzzy subgroups and rings, Honam Math. J. 32(4) (2010), 593-617.

[9] K. C. Lee, H. Kang and K.Hur, Interval-valued fuzzy generalized bi-ideals of a semigroup, Honam Math. J. 33(4) (2011), 603-611.

[10] T.K.Mondal and S.K.Samanta, Topology of interval-valued fuzzy sets, Indian J. Pure Appl. Math. 30(1) (1999), 20-38.

[11] L.A.Zadeh, Fuzzy sets, Inform and Control 8 (1965), 338-353.

[12] _ The concept of a linguistic variable and its application to approximate reasoning-I, Inform. Sci 8 (1975), 199-249.

Jeong Gon Lee

Division of Mathematics and Informational Statistics, and Nanoscale Science and Technology Institute, Wonkwang University,

Iksan 570-749, Korea.

E-mail : jukolee@wku.ac.kr

Kul Hur

Division of Mathematics and Informational Statistics, and Nanoscale Science and Technology Institute, Wonkwang University,

Iksan 570-749, Korea.

E-mail : kulhur@wku.ac.kr

Pyung Ki Lim

Division of Mathematics and Informational Statistics, and Nanoscale Science and Technology Institute, Wonkwang University,

Iksan 570-749, Korea.

E-mail : pklim@wku.ac.kr 